\title{
Grid-Based Multipath with Congestion Avoidance Routing Protocol
}

\author{
Dr. Atul M Gosai ${ }^{1}$,Lakshdeep M Raval ${ }^{2}$ \\ ${ }^{1}$ Associate Professor, ${ }^{2} \mathrm{Ph} . \mathrm{D}$ Scholar, \\ ${ }^{1,2}$ Department of Computer Science, Saurashtra University, Rajkot, Gujarat. India.
}

\begin{abstract}
This Paper gives information about Grid base multipath wireless sensor network. We give a brief introduction about wireless sensor network, how sensor works in this network. How the Routing is done and if there is congestion detected in sensors networks and then how it can be solved. Routing protocols are also classified in several types like Proactive, Reactive, and Hybrid in also given here the details of that. Also here we describe various routing techniques like Traditional and Current techniques. In flooding based techniques, sensor broadcast the information and the destination sensor receives that information. In gossiping techniques, sensor sends information to neighbour sensor and so on. In this paper we also see that if any sensor id dies due to lack of power or any other problems then another path will be routed. Routing protocols are having 3 types Flat routing, Hierarchical routing and Location based routing. In Flat routing SPIN,DD, Rumor routing, etc protocols are used. In hierarchical routing LEACH, PEGASIS, TEEN protocols are used. Now a day LEACH and PEGASIS are widely used in cluster base Wireless Sensor Network. In leach protocol reduced the path using cluster head formulation in this method randomly any sensor make the cluster head $\mathrm{n}$ send messages in the network. In pegasis protocol use chaining concept and find the best path from base station to the destination station. PEGASIS and LEACH provide best routing techniques in wireless sensor network but they also have some disadvantages. We also differentiate flat and hierarchical routing techniques in which case which one gives best performances. Here we introduce the readers to Location based routing protocols GEAR and how it route the path is also briefly described here.
\end{abstract}

Keywords - Wireless Sensor Network protocols, GMCAR, Flooding, Gossiping, SPIN,LEACH, PEGASIS, TEEN

\section{INTRODUCTION}

Wireless sensor networks (WSN) are a specially distributed network that is composed of lots of sensor nodes. It is always deployed in a special area to complete a task of sensing and monitoring. There are many technical challenges associated with sensor networks, such as self-organizing algorithm, energy-efficient routing protocols, data aggregation technology and network lifetime improvements. The energy for sensor networks is very important. It is infeasible to replace battery of sensor. Therefore, conserving energy so as to prolong the network lifetime is becoming one of the key challenges. Recent researches have addressed these topics, such as power-aware channel access, routing and broadcasting, data aggregation protocol and so on $[8,16]$

In this Paper we discuss the how to Sensor Routes the path of destination in wireless network. Traditional Routing techniques like Flooding and Gossiping and also discussed during current routing techniques like Flat routing Techniques, Hierarchical routing Techniques and Location based routing Techniques followed by how congestion is avoided in WSN. Section I is Introduction followed by Section II giving brief about WSN followed by classification of routing protocols and discussing about traditional and current routing algorithms in section III, IV and V. Further, we show our results before conclusion in Section VI followed by Acknowledgement and References in Section VII and VIII.

\section{WIRELESS SENSOR NETWORK}

Wireless grids are wireless computer networks consisting of different category of electronic devices with the ability to share their resources with any other device in the network in an ad hoc comportment. A definition of the wireless grid can be given as: "Ad hoc, distributed resource-sharing networks between Different wireless devices. The following key characteristics further clarify this concept:

- No centralized control

- Small, low powered devices

- Heterogeneous applications and interfaces

- Dynamic and unstable users / resources

A wireless sensor network (WSN)autonomous sensors are also keep an eye on physical or environmental conditions, such as temperature, sound, vibration, pressure, motion or pollutants and to cooperatively pass their data through the network to a main Machine. [2]

The WSN is built of "nodes" from a few to several hundreds or even thousands, where each node is connected to one or sometimes several sensors. Every node contains several parts like radio transceiver, micro-controller, electronic circuit and battery for power supply. The cost of sensor nodes is depending on the energy, memory, computational speed and communications bandwidth.

One of the biggest limitations of the wired grid is that clients are forced to be in aunchangingstatic location as the devices they make use of are to be stiff wired to the grid at all times. This also has a negative influence on the flexibility and scalability of the grid; devices can only connect the grid in locations where the prospective chances exists to physically connect the device to the grid $[1,4]$

Here we show in below figure that how sensors work in WSN. 


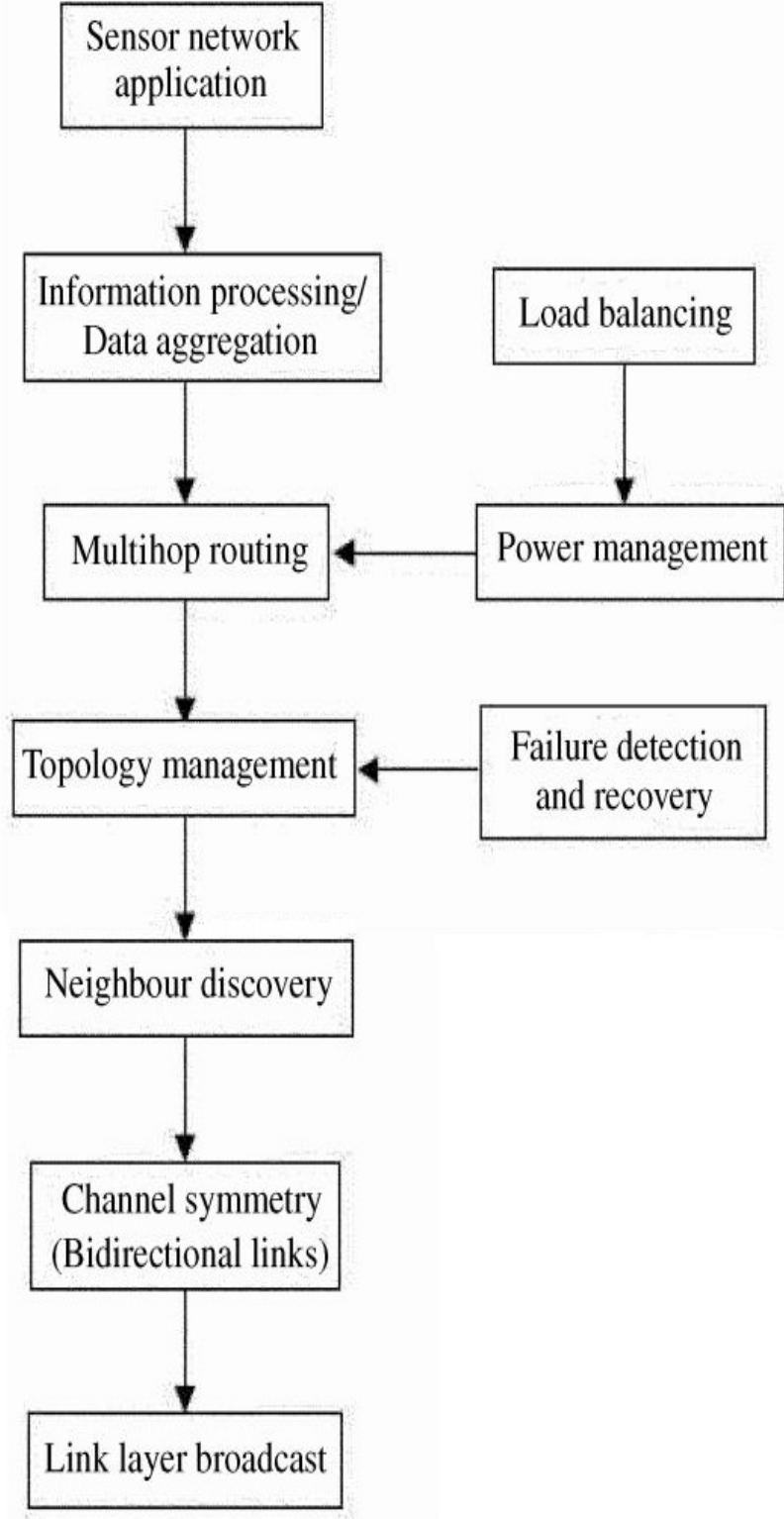

Figure1: Flowchart explaining working of wireless sensor networks.

\section{III.Classification OF Routing Protocols}

Routing protocols are mainly classified in 3 types are as follows:

1. Proactive,

2. Reactive, and

3. Hybrid

Here we Show all the Classification in details.

\section{1) Proactive:}

In a Proactive Protocol the nodes button on their sensors and transmitters, sense the surroundings and convey the information to a BS through the predefined direction. e.g. The Low Energy Adaptive Clustering hierarchy protocol $(\mathrm{LEACH})$ utilizes this sort of protocol.

\section{2) Reactive :}

If there are impulsive changes in the sensed attribute away from some pre-determined threshold value, the nodes instantly react. This type of protocol is used in instance critical applications. e.g. The Threshold sensitive Energy
Efficient sensor Network (TEEN) is an example of a reactive protocol.

\section{3) Hybrid:}

Hybrid protocols integrate both proactive and reactive concepts. They first compute the entire available routes and then improve the routes at the time of routing. e.g. Adaptive Periodic TEEN(APTEEN) is this type of protocol.

\section{IV.TRADITIONAL ROUTING TECHNIQUES}

The Traditional Routing Techniques are also classified as follows:

1. Flooding and

2. Gossiping

Here we Show Both Techniques in details are as follow;

\section{1) Flooding:}

One line of attack is each sensor node broadcast data packet to its neighbours Sensors and this process carry on till the data packet reaches the destination node. If the maximum hop count of the packet is not reached and node itself is not the destination of the packet, this process continues. This technique does not require complex topology maintenance nor route discovery algorithms.

Flooding in presence of all the good points does carry following disadvantages:

1. Implosion:

This is circumstances when replica messages are sent to the same node. This occurs when a node receives carbon copy of the same message from many of its neighbors.

\section{Overlap :}

The same incident may be sensed by more than one node by reason of over lapping of regions of coverage. This results in their neighbors receiving duplicate reports of the same incident.

3. Resource Blindness :

The flooding protocol does not reflect on the available energy at the nodes and results in many surplus transmissions. So it reduces the network lifetime.

\section{2) Gossiping:}

Gossiping is modified version of flooding, where the nodes do not broadcast a packet, but send packets to a randomly selected neighbor. This evades the trouble happening due to Implosion. It takes a long time for a message to propagate throughout the network. Though gossiping has considerably lower overhead than flooding, it does not give any assurance that all nodes of the network will receive the message. It relies on the random neighbor selection to in due course propagate the message throughout the network.

The Sensor node transmits the data packet to a randomly chosen neighbor which in turn selects an additional random neighbor until the destination node is reached.

\section{Current Routing Techniques}

In Traditional Routing Techniques there are quite a few Drawbacks similar to an Implosion, overlap of packets, resource Blindness etc. To overcome all the disadvantages of the Traditional Routing techniques other routing techniques 
and algorithm need to beintroduced and developed that will make use of a dynamic path and generate dynamic routing table in accordance with the density of network traffic and will be capable of finding the best path.

Current Routing Techniques makes use of Classical mechanisms to relay data in sensor networks without the need for any routing algorithms and topology maintenance. Current Routing Techniques which are used in now a days for Routing in Networks are classified as follows:

\section{Flat Routing}
a. SPIN (Sensor Protocols for Information Via Navigation),
b. DD (Directed Diffusion), and
c. Rumor Routing

2. Hierarchical Routing
a. LEACH (Low Energy Adaptive Clustering Hierarchical ) protocols,
b. PEGASIS (Power Efficient Gathering In Sensor Information System) Protocols,
c. TEEN or APTEEN (Threshold-Sensitive Energy Efficient) Protocols.

3. Location Based Routing
a. GEAR (Geographical and Energy Aware Routing) Protocols,
b. GEM

Now we give details about the working of all protocols:

1. Flat Routing:

Flat routing protocols are primarily those protocols that don't work under a predefined network layout and parameter. They facilitate the delivery of packets among routers through whichever available path without considering network hierarchy, distribution and composition.

In the case of Flat Routing Techniques protocols, if any node needs to transmit data, it first searches for a valid available route to the Base Station and then transmits the data. Nodes around the base station may drain their energy quickly during this process.

Here we discuss some Flat Routing Protocols, that are as follows:

\section{a. SPIN:}

SPIN stands for Sensor Protocols for Information via Navigation. SPIN belongs to a family of adaptive protocols also known as Sensor Protocols for Information via Negotiation. It assigns a high-level name to completely describe their collection of information called meta-data.

SPIN has three types of messages: ADV,REQ, and DATA. A sensor node broadcasts an ADV containing meta-data describing actual data. If a neighbor is interested in the data, it sends REQ for the data. Only then the sensor node sends the actual DATA to the neighbor. The neighbor again sends ADVs to its neighbors and this process continues to disseminate the data throughout over the network. The simple version is shown in figure given below in figure 2 .

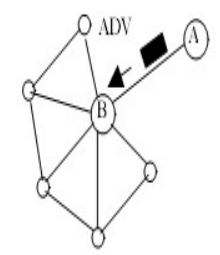

(a)

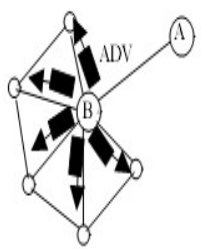

(d)

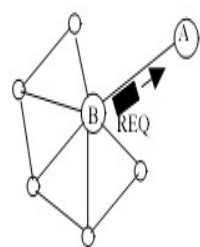

(b)

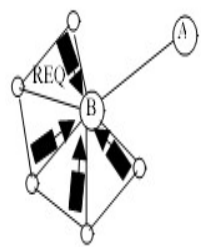

(e)

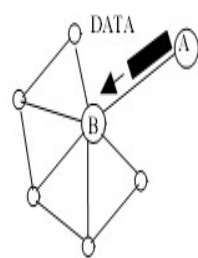

(c)

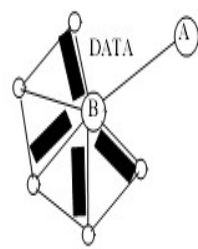

(f)
Figure 2: Data passing strategy in SPIN Routing Techniques.

In SPIN protocols topological changes are confined to a small area. SPIN protocol provides supplementary energy savings than flooding, and metadata negotiation more or less halves the redundant data. The main Drawback of SPIN is data advertisement mechanism cannot guarantee reliable delivery of data.

b. DD: Direct Diffusion.

DD protocols stands for Directed Diffusion.This protocol is helpful in scenario where the sensor nodes themselves generate requests or queries for data sensed by other nodes, instead of all queries arising only from a Base station. Hence the sink for the query could be a BS or a sensor node. The direct diffusion routing protocol improves on data diffusion using interest gradients. Each sensor node names its data with one or more attributes and other nodes express their interest depending on these attributes. Attribute value pairs can be used to describe an interest in intrusion data.

Directed diffusion differs from SPIN in two aspects.

i. Query method

ii. Communication Method.

In Directed diffusion Techniques data can be send by 3 ways are as follows:
i. Propagate Interest
ii. Set up Gradients
iii. Through Reinforcement 


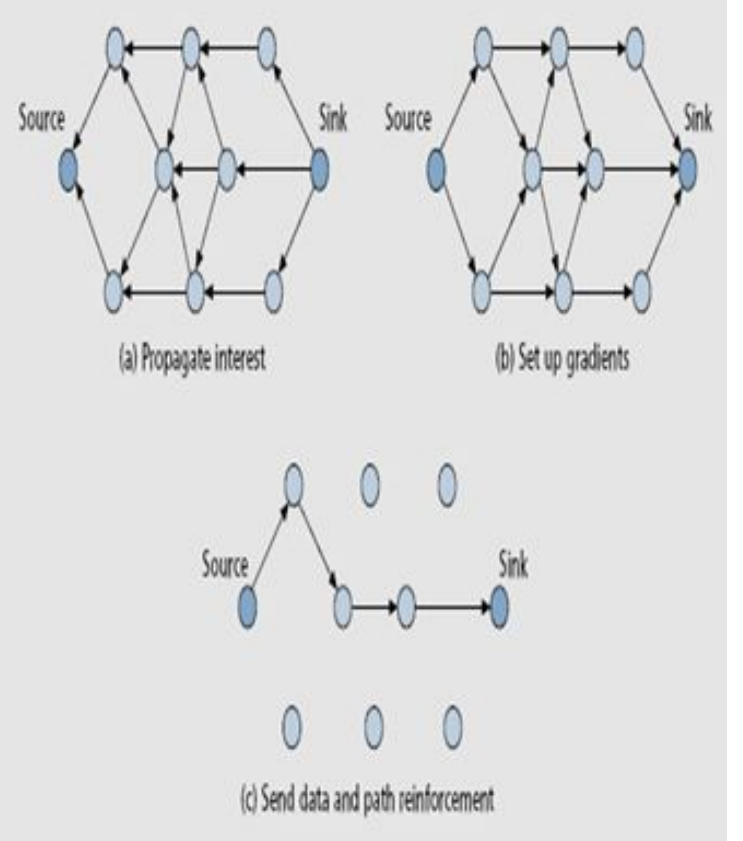

Figure 3: Data passing procedure in DD Routing Techniques.

Directed diffusion may not be applied to all the applications (e.g., environmental monitoring).In Direct Diffusion Techniques Matching data to queries might require some extra overhead.

\section{c. Rumor Routing:}

Rumor Routing is an agent supported path creation algorithm. Agents are long-lived entities created at random by nodes. These are basically packets which are circulated in the network to establish shortest path to entities that they encounter across the network. They can also perform path optimizations for connection with the nodes they visit. When agent finds a node whose path to an event is longer than its own, it updates the nodes routing table.

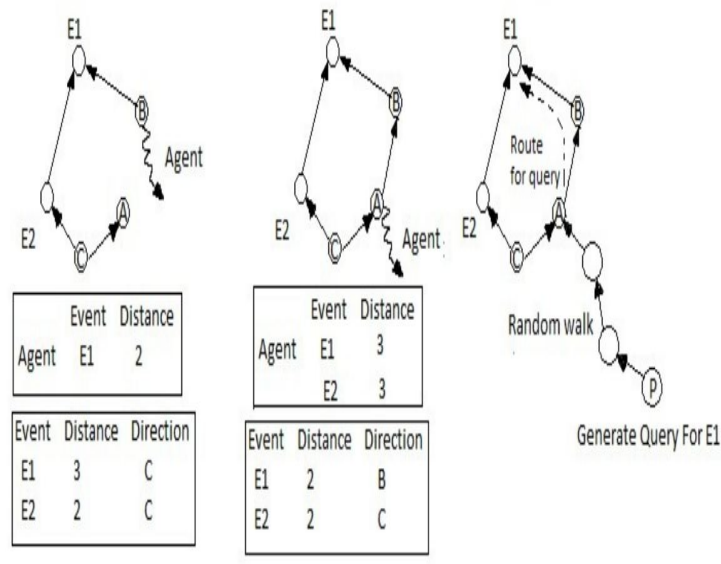

Figure 4: The way Data pass in Rumor Routing Techniques.

\section{Hierarchical Routing:}

Hierarchical routing is efficient to execute energy efficient routing, i.e., higher energy nodes can be used to route and send the information; low energy nodes are used to perform the sensing in the area of interest [10].Hierarchical Routing Techniques are classified as follows: a. LEACH:

LEACH stands for Low Energy Adaptive Clustering Hierarchical protocols. LEACH is the earliest proposed singlehop clustering routing protocol in WSN. It can save the network energy greatly compared with the non-cluster routing algorithm. A lot of other clustering algorithms are projected based on LEACH, such as TEEN (Threshold Sensitive Energy Efficient Sensor Network Protocol), PEGASIS(Power Efficient Gathering in Sensor Information

Systems),HEED (Hybrid Energy-Efficient Distributed Clustering) and so on.[15]

In LEACH code of behavior, all clusters are self-organized; where every cluster holds back a cluster-head and several nonclusters head nodes. Cluster-head node drinks more energy than non-cluster head nodes. With the intention of balancing network energy expenditure and prolonging the network life cycle, it selects cluster head randomly and each node has an equal chance to be cluster-head. The cluster constitution update continuously in operation and one updating process is called a round. The cycle of each round contains two stages: set-up phase and steady-state phase, set-up phase is the establishment phase of a cluster; steady-state phase is the stable data transfer phase.

Set-up phase each node generates a random number between 0 to 1 , and compares this number with the threshold value $T(n)$ .If the number is less than $\mathrm{T}(\mathrm{n})$, the node is selected as a cluster-head.[2]

Once the cluster-head is determined, the cluster-head sends a broadcast message to the network, announced itself as the cluster head; each normal node make a decision which cluster to unite with, in according to the signal strength of the received message, sends a request message to the corresponding clusterhead. The cluster-head receives all the messages sent by the nodes that would like to join in the cluster, confirms them as members of the cluster, then joins them in the routing table and allocates TDMA table of slots for the cluster members telling each member at what time it can transmit data.[3,9,11]

A LEACH protocol is all about progress towards enhancement of node energy and position information to improve the LEACH algorithm, proposes energy balanced clustering algorithm named L-LEACH and LEACH-C.

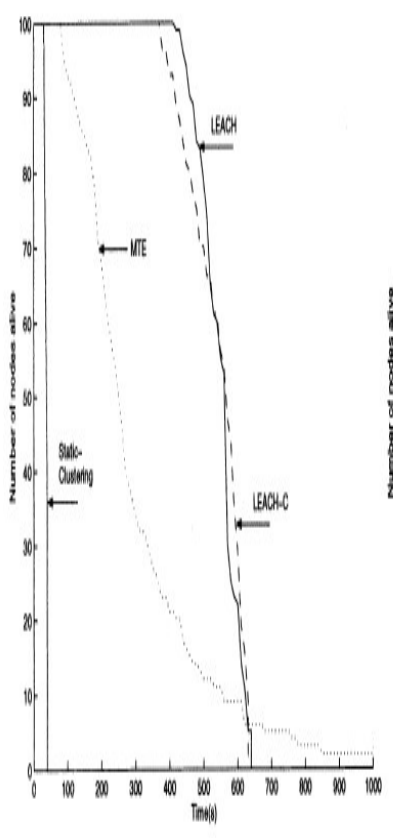

(a)

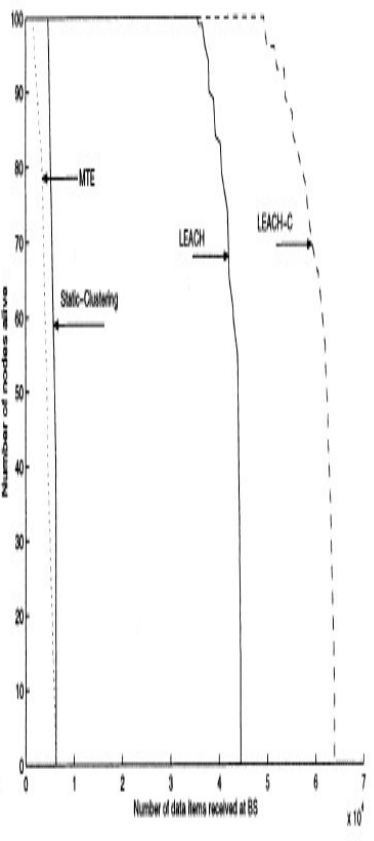

(b) 
Figure 5: Diff between LEACH And LEACH-C.

In above figure we can see that LEACH distributes more data per unit energy than MTE. LEACH-C delivers 40\% more data per unit energy than LEACH.LEACH and LEACH-C's nodes lifetime is much longer than MTE's. LEACH can deliver 10 times the amount of effective data to the BS for the same number of node deaths[5].

\begin{tabular}{llll} 
& SPIN & LEACH & $\begin{array}{l}\text { Directed } \\
\text { diffusion }\end{array}$ \\
\hline Optimal route & No & No & Yes \\
Network lifetime & Good & Very good & Good \\
$\begin{array}{l}\text { Resource } \\
\text { awareness }\end{array}$ & Yes & Yes & Yes \\
Use of meta-data & Yes & No & Yes
\end{tabular}

DD.

Figure 6: Comparison between SPIN,LEACH and

LEACH protocol has a relatively good function in energy consumption through dynamic clustering, keeps the data transmission in cluster which reduces the energy consumption by communicating directly between nodes and the base station, but there are still a lot of inadequacies. The LEACH protocol uses the mechanism of cluster-head rotation, elects clusterhead randomly, after several rounds of data transmission, the residual energy of the nodes will have a great difference, cluster-head or the nodes which are far from the base station will consume more energy. When transmitting data of the same length relatively, if they are selected as cluster-heads after that, they will run out of energy and become invalid. Once the number of invalid nodes increases, it'll have a great influence in the network performance and shorten the life of the network. Cluster member nodes select the optimal cluster-head based on the received signal intensity to join in; do not consider the distance from the node itself to the base station, but considers the distance to be covered between cluster-head and the base station. So normal node may chose the cluster-head that is far away from base station as its optimal cluster-head, this not only is the heavy burden to the cluster-head but also increases the extra energy consumption, which is not beneficial to balance network energy consumption. The protocol assumes that all nodes begin with the same quantity of energy capacity in each election round, assuming that being a $\mathrm{CH}$ consumes approximately the same amount of energy for each node.[7,9]

\section{b. PEGASIS:}

PEGASIS is stands for Power-Efficient Gathering in Sensor Information Systems. It is an algorithm based on chain, which uses greedy algorithm to form data chain. Each node aggregates data from downstream node and sends it to upstream node along the chain.[15, 16]

PEGASIS has some advantages when compared with LEACH: It eradicate the overhead in dynamic formation of cluster; the distance between nodes on chain is much shorter than from nodes to cluster heading LEACH and so on. Therefore, PEGASIS can save much energy. In PEGASIS, each node communicates only with a close neighbor and takes turns transmitting to the base station, thus reducing the amount of energy spent per round. Simulation results show that PEGASIS performs better than LEACH by about $100 \%$ to
$300 \%$ when $1 \%, 20 \%, 50 \%$, and $100 \%$ of nodes die for different network sizes and topologies.

Although PEGASIS has better performance, it also has a shortcoming: using greedy algorithm to construct chain, which will result in distance between a pair of sensors that becomes too long. In this condition, this pair of sensors will consume much energy than other sensors in transmitting data phase. Thus, they may probably die early.[12]

Chain construction to construct the chain we start from the farthest node from the base station, and then greed approach is used to construct the chain.

Leader of each round is selected randomly. If $\mathrm{N}$ is number of node as selected as head node for some round randomly selecting head node also provides benefit as it is more likely for nodes to die at random locations thus providing robust network. When a node dies chain is reconstructed to bypass the dead node.Head node receives all the fused data and sends to the BS.[13]

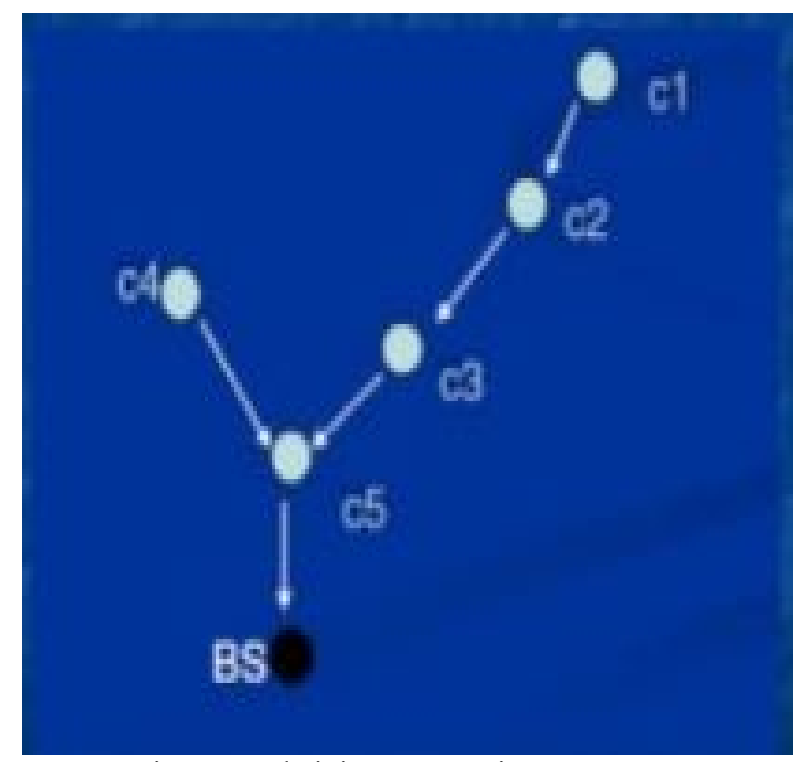

Figure 7: Chaining Process in PEGASIS

In chaining process, a node will consider average distance of formed chain. If the distance from neighboring node to its upstream node is longer than distance thresh (the distance thresh can obtain from average distance of formed chain), the closest node is a "farnode". If the closest node joins the chain, it will emerge as a "long chain". In this condition, the "far node" will search a nearer node over the formed chain. Through this method, the new protocol EB-PEGASIS can avoid "long chain" effectively. It not only save energy on sensors, but also balance the energy consumption of all sensornodes.

In below Image we can See that the Percentage of nodes demise during the transmission. This is a Performance result for $100 \mathrm{~m} * 100 \mathrm{~m}$ Network with initial energy $5 \mathrm{~J} /$ Node. 


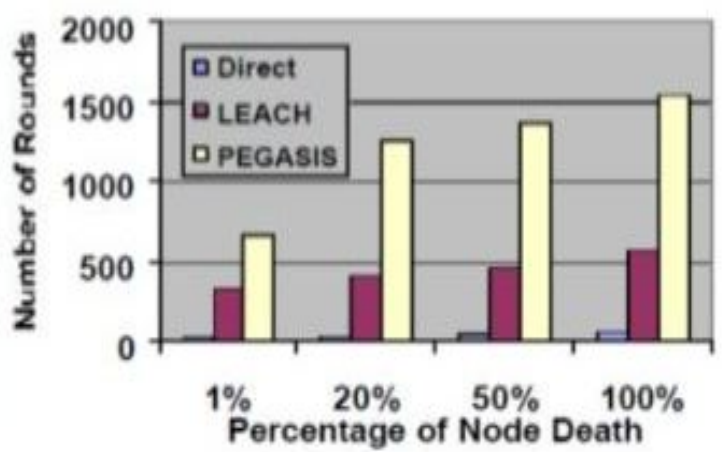

Figure 8: Performance chart of nodes death in different Protocols.

The Disadvantages of PEGASIS protocol is only that PEGASIS assumes each sensor node is competent to communicate with the BS directly. We assume that all sensor nodes have the same level of energy and are likely to die at the same time [16].

\section{c. TEEN:}

TEEN is stands for Threshold-Sensitive Energy Efficient Protocols. TEEN'S CH sensor sends its members a hard threshold and a soft threshold. TEEN'S appropriateness for time-critical sensing applications is admirable. TEEN is also quite resourceful in terms of energy consumption and response time. TEEN also allows the user to manage the energy consumption with precision to suit the application.

In below figure we show comparison of other protocols with TEEN protocols in the terms of Energy verses number of Nodes.

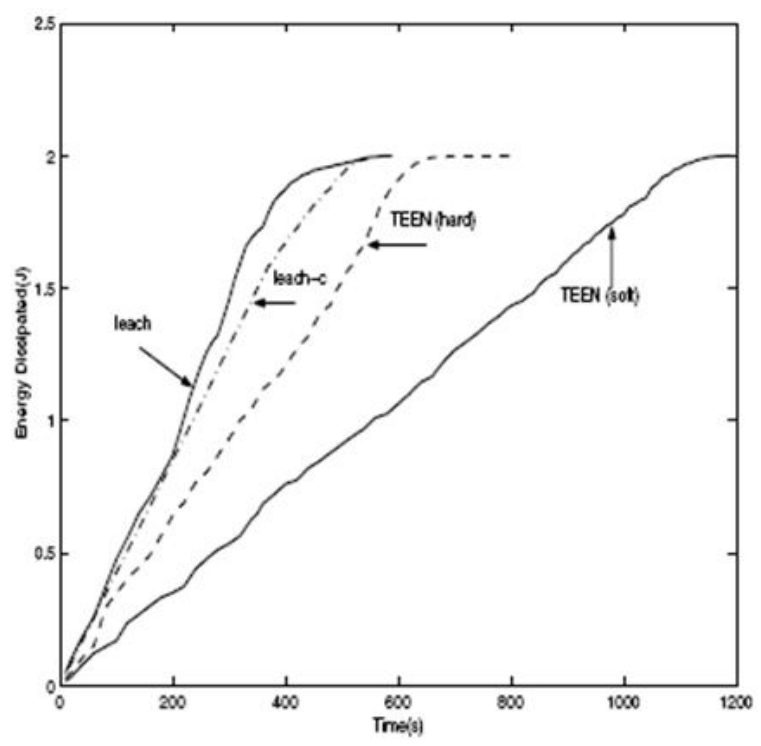

Figure 9: Comparison of all protocols in term of energy they used.

In above figure we show that the TEEN consume awfully less energy in contrast to the LEACH and LEACH-c Protocols. So the Life of nodes and network will be extended and performance will be in good health.

In below figure we can see that Compare to Other Protocols in TEEN nodes can alive for long time because in TEEN protocols Energy is not wasted by nodes.

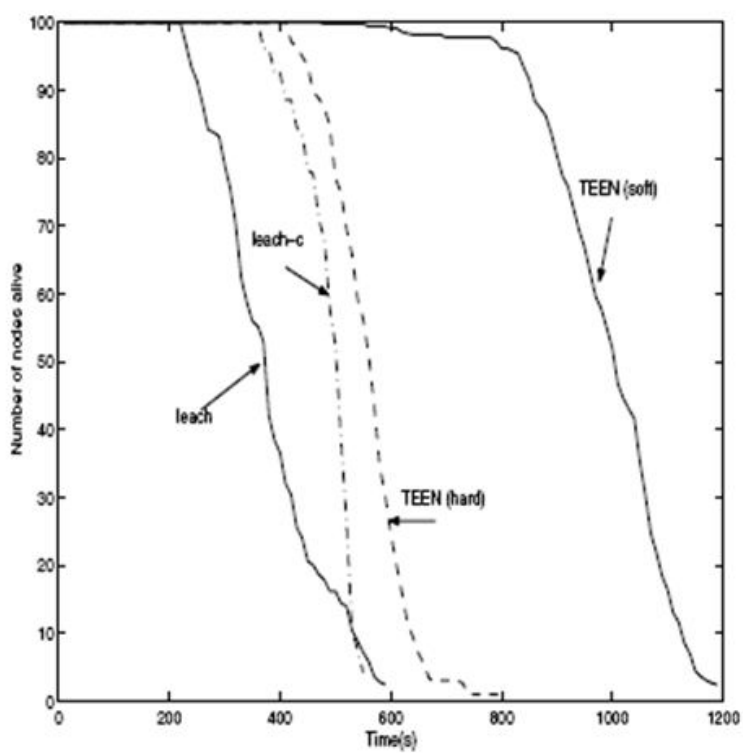

Figure 10: Comparison of all protocols in term of Nodes are alive.

In below table the differences between flat Routing and Hierarchical routing techniques are given.

\begin{tabular}{|c|c|}
\hline Flat Routing & Hierarchical Routing \\
\hline Contention-based routing & reservation-based scheduling \\
\hline Collision overhead present & Collisions avoided \\
\hline $\begin{array}{l}\text { Variable duty cycle by } \\
\text { controlling sleep time of nodes }\end{array}$ & $\begin{array}{l}\text { Reduced duty cycle due to } \\
\text { periodic sleeping }\end{array}$ \\
\hline $\begin{array}{l}\text { Node on multi hop path } \\
\text { aggregates incoming data from } \\
\text { neighbours }\end{array}$ & $\begin{array}{l}\text { Data aggregation by } \\
\text { clusterhead }\end{array}$ \\
\hline $\begin{array}{l}\text { Routing can be made optimal } \\
\text { but within added complexity }\end{array}$ & $\begin{array}{l}\text { Simple but non-optimal } \\
\text { routing }\end{array}$ \\
\hline $\begin{array}{l}\text { Links formed on the fly without } \\
\text { synchronization }\end{array}$ & $\begin{array}{l}\text { Requires global and local } \\
\text { synchronization }\end{array}$ \\
\hline $\begin{array}{l}\text { Routes formed only in regions } \\
\text { that have data for transmission }\end{array}$ & $\begin{array}{l}\text { Overhead of cluster formation } \\
\text { throughout the network }\end{array}$ \\
\hline $\begin{array}{l}\text { Latency in waking up } \\
\text { intermediate nodes and setting } \\
\text { up the multipath }\end{array}$ & $\begin{array}{l}\text { Lower latency as multiple } \\
\text { hops network formed by } \\
\text { cluster-heads always available }\end{array}$ \\
\hline $\begin{array}{l}\text { Energy dissipation depends on } \\
\text { traffic patterns }\end{array}$ & Energy dissipation is uniform \\
\hline $\begin{array}{l}\text { Energy dissipation adapts to } \\
\text { traffic pattern }\end{array}$ & $\begin{array}{l}\text { Energy dissipation cannot be } \\
\text { controlled }\end{array}$ \\
\hline Fairness not guaranteed & Far channel allocation \\
\hline
\end{tabular}

3. Location-based routing:

Location based routing protocols need some location information of the sensor nodes. Location information can be obtained from GPS (Global Positioning System) signals, 
received radio signal strength, etc. Using location information, an optimal path can be formed without using flooding techniques.Location Based Routing algorithms are classified as follows:

\section{a. GEAR:}

GEAR stands for Geographic and Energy Aware Routing protocols. The key scheme is to confine the number of interests in directed diffusion by only considering a certain region rather than sending the interests to the whole network. GEAR protocol keeps an estimated cost and a learning cost balanced and under the edge.

\section{b. GPRS:}

One another Protocols are GPSR, which is designed for general mobile ad hoc networks. It has two parameters, Uniform Traffic and Non-uniform Traffic. For uneven traffic distribution, GEAR delivers $70-80$ percent more packets than GPSR. For uniform traffic pairs GEAR delivers 25-35 percent more packets than GPSR.[14]

\section{VI.CONCLUSION}

In this research we have described the Grid base multipath congestion avoidance protocols where, we also discussed different types of protocols that are used in wireless sensor network for routing and avoiding congestion. We also shown, how it will pick the finest path for transporting the packet data from numerous routes. We also described protocols that will choosesuperlative path for sending data from base station to destination other than normally opted. In this paper we also compared all the protocols on the basis of common aspect of the power consumption and energy draining behavior. We gave comparative analysis based on the way nodes are used and the remaining energy left before nodes die because of issues like instability, delay, interference and continuous scanning for the networks.

\section{REFERENCES}

[1] Robert AklandUttarasawant, Grid-based Coordinated Routing in Wireless Sensor Networks.

[2] M.J.Handy,M.Haase,D.Timmermann,Low Energy Adaptive Clustring Hierarchy with Deterministic Cluster-Head Selection

[3] QianLiao,HaoZhu,An Energy Balanced Clusterin Algorithm Based on LEACH Protocol

[4] Mohammad asumuzzamanBhuiyan,IqbalGondalJoarderKamruzzaman,Location Aided Congestion Awae Routing in Wireless Sensor Networks

[5] MortazaFahimiKhatonAbad,Mohammad Ali jabraeiljamali,Modify LEACH Algorithm for Wireless Sensor Network

[6] Majid I. Khan,Congestion Avoidance and Energy Efficient Routing Protocol for Wireless Sensor Networks with a Mobile Sink

[7] JaewonKang,BadriNath,YanyongZhang,Shengchaoyu, Adaptive Resource Control Scheme to Alleviate Congestion in Sensor Netwotks

[8] Lucian Popa,CostinRaiciu,IonStoica,David S. Rosenblum, Redusing Congestion Effects inWireless Networks by Multipath Routing

[9] Mark A.Perillo,wendiB.Heinzelman, Wireless Sensor Network Prorocols

[10] Bharat Bhargava, Self-Adjusting Congestion Avoidance Routing Protocol for Ad-Hoc Networks

[11] SinemColeriErgen and PravinVaraiya, TDMA Scheduling Algorithms for Wireless Sensor Networks

[12] LindseyS:PEGASIS,comput.Syat.Res.Dept.Aeroap.Corp.Angeles.CA.US A.IEEE Xplore

[13] Jayashree A,G. S. Biradar,V.D.Mytri, Review of Multipath Routing Protocols in Wireless Multimedia Sensor Network - A Survey

[14] N.NarasimhaDatta and K.Gopinath, A survey of routing algorithms for wireless sensor networks

[15] Al-Karaki, J. N. and A. E. Kamal, "Routing Techniques in Wireless Sensor Networks: A Survey", IEEE Wireless Communications, Volume 11, No. 6, Dec. 2004, pp. 6--28.

[16] Shagan Paul Kaur, Mrs.Amandeep Kaur, Review of Power-Efficient Gathering in Sensor Information System(PEGASIS) for Network Optimization in WSN, International Journal of Advanced Research in
Computer Science and Software Engineering (IJARCSSE), Volume 4, Issue 6, June 2014, Pg no. 872 - 877. 